\title{
Holographic reconstruction technology used for intraoperative real-time navigation in robot-assisted partial nephrectomy in patients with renal tumors: a single center study
}

\author{
Shaohua Zeng ${ }^{1, \# \wedge}$, Yu Zhou ${ }^{3 \#}$, Min Wang ${ }^{3}$, Hui Bao ${ }^{3}$, Yanqun $\mathrm{Na}^{4}$, Tiejun $\mathrm{Pan}^{3}$ \\ ${ }^{1}$ The First School of Clinical Medicine, Southern Medical University, Guangzhou, China; ${ }^{2}$ Department of Urology, The Sixth Affiliated Hospital of \\ Guangzhou Medical University, Qingyuan, China; ${ }^{3}$ Department of Urology, General Hospital of the Central Theater Command, Wuhan, China; \\ ${ }^{4}$ Department of Urology, Peking University Shougang Hospital, Peking University Health Science Center, Beijing, China \\ Contributions: (I) Conception and design: Y Na, T Pan; (II) Administrative support: None; (III) Provision of study materials or patients: S Zeng, Y \\ Zhou; (IV) Collection and assembly of data: M Wang, H Bao; (V) Data analysis and interpretation: S Zeng, Y Zhou; (VI) Manuscript writing: All \\ authors; (VII) Final approval of manuscript: All authors. \\ \#These authors contributed equally to this work. \\ Correspondence to: Tiejun Pan. Department of Urology, General Hospital of the Central Theater Command, Wuhan, China. Email: mnwkptj@aliyun.com.
}

Background: To explore the efficacy and advantages of real-time navigation using holographic reconstruction (HR) technology combined with da VinciTM robotic system for partial nephrectomy $(\mathrm{PN})$ in patients with renal tumor.

Methods: The clinical data of 41 patients with totally intrarenal tumors receiving robot-assisted partial nephrectomy (RAPN) from April 2018 to October 2020 in our department were collected and retrospectively analyzed. All operations were performed by the same surgeon. HR technology and three-dimensional (3D) reconstruction techniques were applied for real-time navigation to resect tumors using the da VinciTM robotic system. The relevant clinical parameters and surgical outcomes of the patients were recorded and analyzed.

Results: HR technology allowed accurate evaluation of tumors, renal hilus vessels, and surrounding organs during the operation. With real-time navigation HR, all cases were performed by RAPN. The mean operative time was $115.3 \pm 20.3$ (range, 70-153) minutes, and the warm ischemia time (WIT) was 18.7 \pm 3.9 (range, 13-28) minutes. The estimated blood loss (EBL) was 98.8 \pm 18.7 (range, 60-141) mL. Negative surgical margins were reported in all cases. Patients with absence of grade $\leq 1$ Clavien-Dindo complications. Compared with the clinical outcomes of standard RAPN, as reported in the literature, HRassisted technology reduced the mean operative time, the WIT, and the EBL in patients undergoing RAPN. Therefore, combining HR with robotic abdominal surgery can enhance the efficiency of locating blood vessels and allow for more accurate resection of tumors.

Conclusions: As a novel and promising computer digital technology, HR can significantly improve the success of RAPN operations. This retrospective study demonstrated that HR-assisted operations resulted in shorter operation times and less perioperative complications and were thus safer and more effective in patients with renal tumors compared with RAPN not used HR.

Keywords: Holographic reconstructions (HRs); renal cell carcinoma; real-time navigation; robot-assisted partial nephrectomy (RAPN)

\footnotetext{
$\wedge$ ORCID: 0000-0001-8660-2317.
} 
Submitted May 13, 2021. Accepted for publication Jul 13, 2021.

doi: $10.21037 /$ tau-21-473

View this article at: https://dx.doi.org/10.21037/tau-21-473

\section{Introduction}

With advancements in medicine, partial nephrectomy (PN) has become a well-accepted standard for the nephronsparing treatment of pT1 renal parenchymal tumors (1). Technological advances have fueled a paradigm shift from open surgery to minimally invasive techniques for PN (2). Indeed, minimally invasive approaches, including laparoscopic PN and more recently, robot-assisted (RA) procedures, have been increasingly accepted as a reliable surgical option for patients with small renal tumors, largely due to the refinement of surgical techniques and instruments $(3,4)$.Compared with open nephron-sparing surgery (NSS), robot-assisted partial nephrectomy (RAPN) has achieved good results with accurate resection of tumors, protection of renal function, and prevention and control of perioperative complications (5-7). These advantages have resulted in a surge of robotic surgeries in hospitals.

The concepts of "precision medical treatment" and "precision surgery" have also attracted considerable attention. With continuous developments in the field of medical imaging, intraoperative guidance for the precise excision of tumors has become a new direction in laparoscopic surgery (8). Virtual reality technology uses computer simulation systems to generate three-dimensional (3D) dynamic images which integrate with entity behaviors and object interactions to create a "real scene" that allows user immersion and interaction. Holographic reconstructions (HRs) technology is a successful virtual reality technology that combines a full and immersive experience of threedimensionality, interactivity, and versatility. Significantly, the realistic model allows the user to appreciate the detailed anatomy at a glance. The patient's computed tomography angiography (CTA) images are transformed into a holographic digital virtual organ to clearly display the internal kidney structures, providing real-time separation, interaction, measurements, and analytical functions. This assists doctors accurately identify and locate blood vessels, surrounding organs, and the target tumor, which facilitates planning of the operation mode and scope. Furthermore, robot operating systems allows a more magnified view and increased flexibility compared to the human arm. This renders the manipulations easier and safer.
In what was to our knowledge the first report that using the HR technology to navigated RAPN until now. This retrospective study analyzed the efficacy of HR in 41 patients with renal tumors who underwent RAPN with satisfactory results. Here we presented our preliminary experiences with this novel surgical navigation technique. Using this novel HR navigation system, the clinical outcomes of the surgery were significantly improved.

We present the following article in accordance with the STROBE reporting checklist (available at https://dx.doi. org/10.21037/tau-21-473).

\section{Methods}

\section{Patients}

A total of 41 consecutive patients with renal tumors who underwent RAPN in the Department of Urology Surgery, General Hospital of the Central Theater Command, Wuhan, China, between April 2018 to October 2020 were retrospectively included in this study. All operations were performed by the same experienced surgeon. Patient demographics, tumor characteristics, and preoperative clinical data are summarized in Table 1. All patients were preoperatively evaluated using an enhanced thin-slice CTA.

All procedures performed in this study involving human participants were in accordance with the Declaration of Helsinki (as revised in 2013). The study was approved by ethics board of General Hospital of Central Theater Command of the People's Liberation Army (Number: [2018]012) and informed consent was taken from all the patients.

\section{HR technique}

The HR software was produced by Renxin MedTech (Beijing China) and implemented on a NAVIGATOR workstation (Renxin MedTech) consisting of a Windows ${ }^{\circledR}$ based computer with a stereoscopic screen to visualize objects in augmented reality. All patients underwent preoperative CTA examination. The CTA data were collated and saved in DICOM format, and uploaded to the NAVIGATOR workstation (Figure 1A,1B). Using the 
Table 1 Patient demographics, tumor characteristics

\begin{tabular}{lc}
\hline Parameters & $\mathrm{N}(\%)$ \\
\hline Patient demographics & 41 \\
Numbers & $60.1[41-78]$ \\
Ages, year [range] & \\
Gender & $27(65.9)$ \\
Male & $14(34.1)$ \\
Female & $24.7[22.1-28.1]$ \\
Body mass index, kg/m² [range] & \\
Tumor characteristics & \\
Tumor site & $22(53.7)$ \\
Right & $19(46.3)$ \\
Left & \\
Tumor size, cm & \\
$\leq 4$ & $30(73.2)$ \\
$>4$ & $11(26.8)$ \\
R.E.N.A.L. score & \\
$4-6$ & \\
$7-9$ & \\
Location & \\
Hilar & $(36.3)$ \\
\hline & \\
Others & \\
\hline
\end{tabular}

reconstruction and virtual image technology, the abdominal organs, the kidneys and their volume, the position and blood supply of the tumor, and other information can be accurately mapped and reconstructed. The reconstructed images and solid viscera can be mixed to achieve translucent, clear real-time navigation displays (Figure 1C).

\section{Real-time navigation and surgical techniques}

The whole workstation can be easily relocated to the operating room prior to surgery. The display of the HR workstation is synchronized with the TilePro multi-input display (Intuitive Surgical Inc., Sunnyvale, CA, USA). The HR renders and fuses the collected information into realtime abdominal contents. When the HR system is activated, two digital video interfaces will appear in the TilePro multi- input display, one is the real-time larger image displayed by the laparoscopic camera, the other is the interface of the HR integrated with the virtual reality technology for intraoperative real-time navigation (Figure $2 A$ ). The HR system is manipulated by the assistant surgeon to view specific anatomy. The anatomy model can be rotated, zoomed, hyalinized, obliterated, combined, or split to show organs, lesions, blood vessels, and anatomical relationships (Figure 2B).

After general anesthesia, the patient was placed in the lateral position, and the operation was performed through a transperitoneal approach. The laparoscopic camera was inserted into the abdominal cavity. The colon was dissociated to the midline, and the liver or spleen was further dissociated to completely expose the renal hilum area. With the HR navigation guidance of virtual reality fusion, the upper ureter, the renal artery, and the renal vein were dissociated, and the blood vessels were sequentially clamped using bulldog clamps. However, (17 out of 41 cases) The tumor was accurately located and seconddissociated (Figure 3), and the artery supplying the tumor was clamped by bulldog clips in a zero warm ischemia manner. The 360 panoramic view of the tumor, including the margin between the tumor and the renal parenchyma, is shown by HR in Figure 4. The renal parenchyma nearest to the tumor was selected for the incision, and the tumor was excised along the tumor bed in a reverse manner towards the normal renal parenchyma. In cases where the renal pedicle blood vessels were exposed or close to the renal hilum, the skirt suture or "C" suture method was used (13 out of 41 cases). The collecting system and the large vessels were closed with 3-0 V-Loc sutures, and renal parenchymal sutures using 2-0 V-Loc were then placed for cross-compression along the defect. The assistant inserted an entrapment bag and the tumor was extracted.

\section{Statistical analysis}

Continuous variables are shown as the mean and range. Categorical variables are shown as the frequency and percentage. All statistical analyses were performed using the SPSS 23.0 software (IBM Co., Armonk, NY, USA).

\section{Results}

The operation was successfully completed on all patients without conversion to open surgery or radical nephrectomy due to massive renal hemorrhage. Negative surgical 

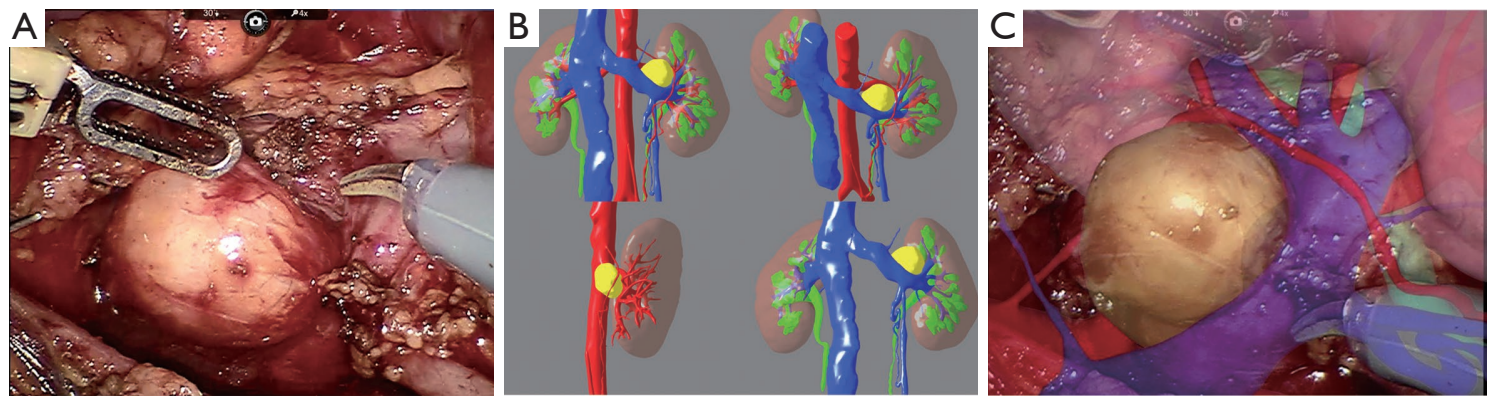

Figure $13 \mathrm{D}$ image reconstruction and mixed reality technology in HR. (A) The live renal tumor image was displayed by the laparoscopic camera; (B) the generate 3D dynamic image was generated by HR in same case; (C) mixed the live renal tumor image and 3D dynamic image to navigated the vessel and tumor margin unexposed. 3D, three-dimensional; HR, holographic reconstruction.
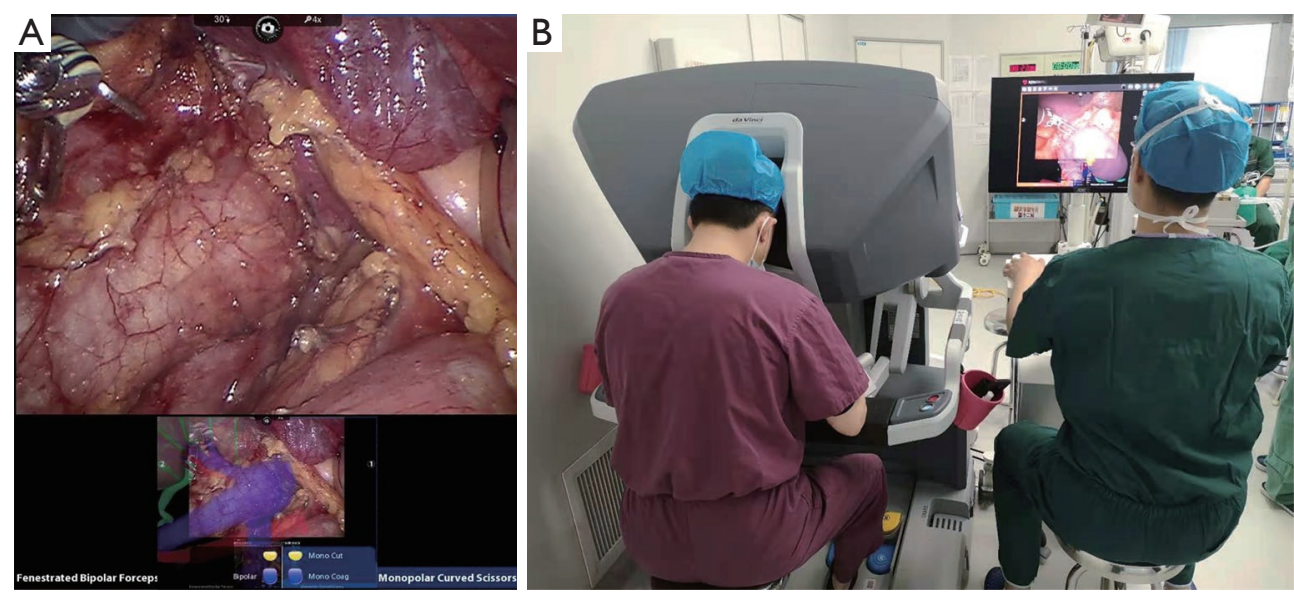

Figure 2 Mixed reality real-time navigation combine surgery technique. (A) The live image was displayed by the laparoscopic camera (large), the mixed reality real-time navigation image (small); two images were appeared in the TilePro multi-input display; (B) HR workstation is synchronized with the TilePro multi-input display the surgeon (dressed in red) manipulated the console, the assistant manipulated the HR workstation. HR, holographic reconstruction.

margins were reported in all cases. No patients required intraoperative or postoperative blood transfusions and none experienced severe complications (Table 2). The clinical characteristics for each patient including final pathology, margin status, and postoperative complications are listed in Table 2. Operation time, warm ischemia time (WIT), estimated blood loss (EBL), preoperative glomerular filtration rate (GFR), and postoperative renal GFR of the impacted kidney at the 3-month follow-up were collated (Table 3). There were a few postoperative complications including abdominal distention, unhealing wounds, and postoperative fever (Clavien classification I), all of which spontaneously subsided with symptomatic treatment. Table 3 shows that the mean operative time was $115.3 \pm 20.3$ (range, 70-153) minutes and the WIT was $18.7 \pm 3.9$ (range, 13-28) minutes. The EBL was 98.8 \pm 18.7 (range, 60-141) $\mathrm{mL}$. The estimated GFR decreased 16\% from pre-operation to 3 months post-operation. Compared to standard RAPN reported in the literature (Table 4) (9-19), the implementation of HR with robotic abdominal surgery effectively reduced the operation time. Real-time intraoperative navigation enabled surgeons to easily identify the blood vessels of the renal hilum, thus avoiding damage to the blood vessels.

\section{Discussion}

A growing number of studies have demonstrated that 

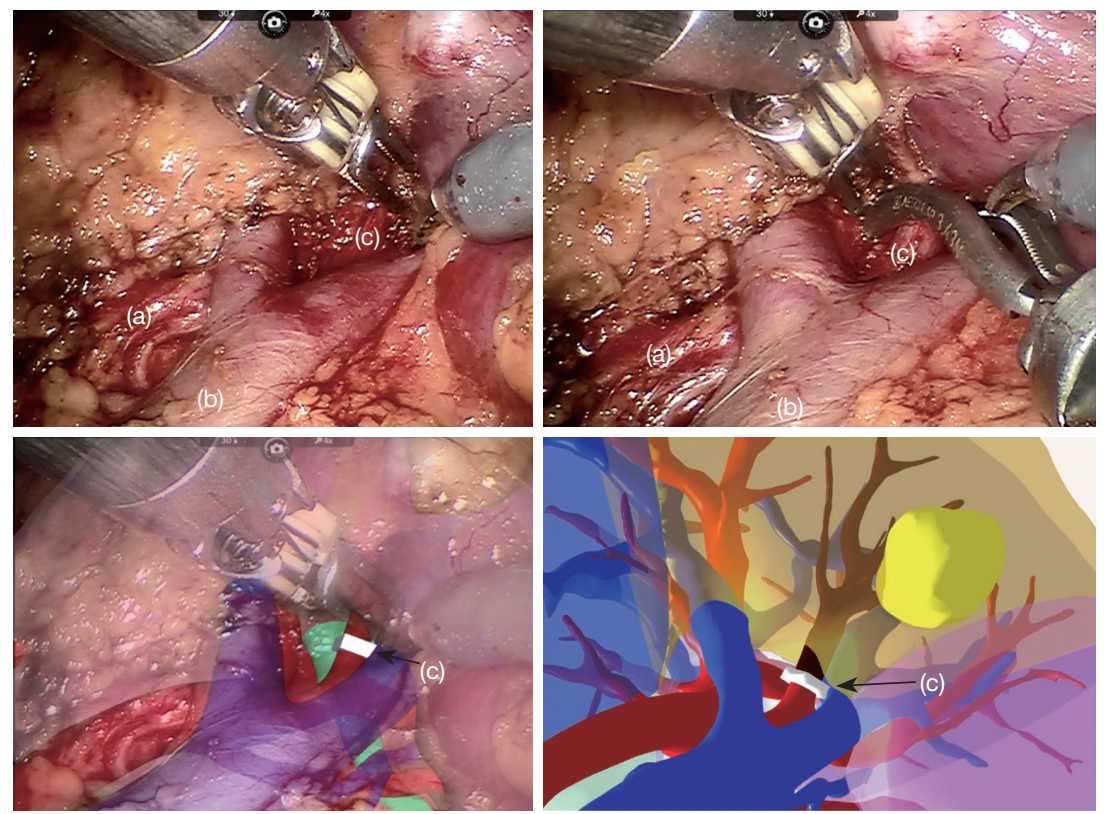

Figure $3 \mathrm{HR}$ real-time navigation used to assist the location and isolation of vessels supplying the tumor. (a) Renal artery; (b) renal vein; (c) tumor supply artery. HR, holographic reconstruction.
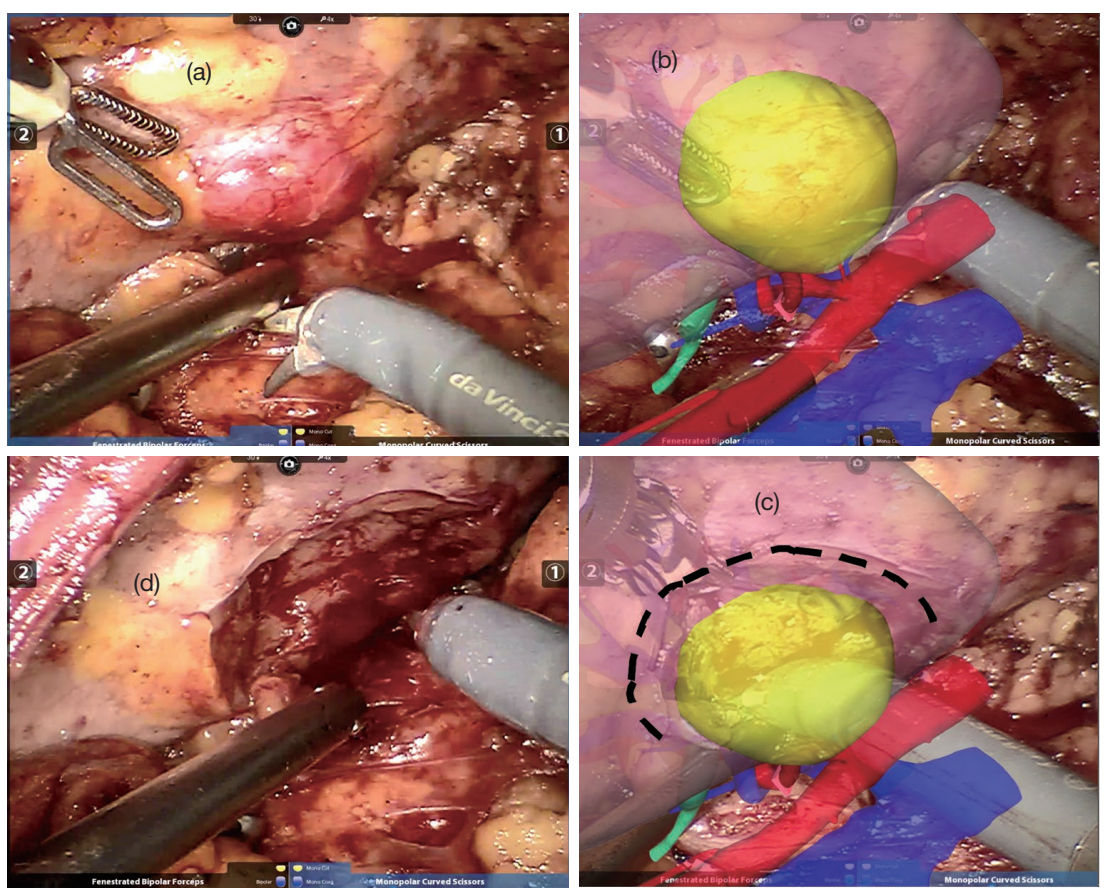

Figure 4 HR real-time navigation assisted to locate and resect endophytic tumor margin. (a) first step: isolate and confirm the tumor approximate location; (b) second step: real-time navigation to visualized the tumor precise margin and vessels by HR; (c) third step: resected the tumor according to the real-time navigation display; (d) fourth step: the complete resection endophytic tumor image. HR, holographic reconstruction. 
Table 2 Pathology, surgical margin status, complications

\begin{tabular}{lc}
\hline Parameters & $\mathrm{N}(\%)$ \\
\hline Final pathology & $35(85.4)$ \\
Clear cell & $5(12.2)$ \\
Chromophobe & $1(2.4)$ \\
Papillary & \\
Perioperative complications & \\
Intraoperation & $0(0.0)$ \\
Conversion to open & $0(0.0)$ \\
Adjacent organ injury & $0(0.0)$ \\
Blood transfusion & \\
Postoperation & $7(17.1)$ \\
Fever & $14(34.1)$ \\
Abdominal distention & $3(7.3)$ \\
Unhealing wound & $0(0.0)$ \\
Blood transfusion & $0(0.0)$ \\
Urine leak & $0(0.0)$ \\
Renal bleed & $0(0.0)$ \\
\hline
\end{tabular}

Table 3 Operation time, WIT, EBL, perioperative GFR

\begin{tabular}{lc}
\hline Parameters & $\begin{array}{c}\text { Mean } \pm \text { standard } \\
\text { deviation [range] }\end{array}$ \\
\hline Operative time, min & $115.3 \pm 20.3[70-153]$ \\
WIT, min & $18.7 \pm 3.9[13-28]$ \\
EBL, $\mathrm{mL}$ & $98.8 \pm 18.7[60-141]$ \\
Perioperative eGFR, mL/min/1.73 m² & \\
Diseased kidney preoperative operation & $35.7 \pm 6.9[27-45]$ \\
Diseased kidney postoperative 3-month & $22.1 \pm 7.2[19-38]$ \\
\hline
\end{tabular}

WIT, warm ischemia time; EBL, estimated blood loss; GFR, glomerular filtration rate; eGFR, estimated GFR.

NSS achieves similar oncological outcomes compared to radical nephrectomy for small renal tumors (20). NSS has been shown to significantly reduce the onset of chronic renal failure compared to radical nephrectomy. Indeed, the increased literature and American Urological Association guidelines advocate the implementation of $\mathrm{PN}$ in clinical stage T1a-T1b renal tumors where preservation of the renal parenchyma is recommended $(21,22)$. For NSS, the advent of laparoscopic techniques, together with advancements in instrumentation and imaging, have led to improvements in precision renal tumor resections, which can maximally preserve the renal parenchyma and renal function (23). RA laparoscopic techniques have the following advantages: (I) $3 \mathrm{D}$ images of the operative field can be visualized with the naked eye; (II) the robotic arm does not fatigues, does not shake, and is equipped with 360 degrees of free rotation; and (III) the laparoscopic camera can be freely controlled by the surgeon. Thus, RA laparoscopic surgery is considered the second most important technical innovation after laparoscopic techniques (24).

Mixed reality technology, including augmented reality and virtual reality, is a new breakthrough in imaging technology. New visualization environments can be created by introducing virtual information into real-life scenarios, resulting in the coexistence of physical and digital objects and allowing for real-time interactions. Mixed reality technology enables precision surgical operations in all segments of surgical treatment (25). HR is a wellestablished and successful area of mixed reality technology $(8,26)$. HR technology combined with RA laparoscopic systems can enhance surgical precision, resulting in less intraoperative bleeding, fewer postoperative complications, and faster rehabilitation for patients.

In our department, we found HR to be more exhaustive and precise compared to CTA when assessing the morphological location of the tumor. Visualizing the relationship between the blood vessels supplying the tumor and other renal arteries and vein was beneficial to developing the surgical plan and anatomical approach. The HR data also provided insights into possible difficulties of the surgery and possible intraoperative complications. In addition, the HR created $3 \mathrm{D}$ images of the tumor, blood vessel, kidneys, and other organs on the computer screen, and this was a useful tool during preoperative communications and discussions with the patients. Patients were able to gain a deeper understanding of the surgical modalities and content (Figure 1B), as well as the risks and possible postoperative complications. All this is important for improving doctor-patient relationships and patient compliance.

Real-time intraoperative navigation with $\mathrm{HR}$ is a revolutionary improvement and innovation in surgical operations. Previously, operations in traditional surgery procedure were performed on the basis of what could be 
Table 4 Clinical data of patients treated with RAPN—a review of the published literature

\begin{tabular}{|c|c|c|c|c|c|c|c|}
\hline Authors & Numbers & Time (min) & Size (mm) & PSM (\%) & WIT (min) & $\mathrm{EBL}(\mathrm{mL})$ & eGFR DR (\%) \\
\hline Michael W. Patton & 90 & 196 & NR & 0 & NR & 150 & 11 \\
\hline Agnes J. Wang & 40 & 140 & 25 & $1 / 40(2.5)$ & 20 & 136 & NR \\
\hline Brian M. Benway & 129 & 189 & 28 & $5 / 128(3.9)$ & 19.7 & 155 & NR \\
\hline Daisuke Motoyama & 65 & 180 & 23 (median) & 0 & 15 (median) & 50 (median) & 9.8 \\
\hline Jonathan S. Ellison & 150 & 160 & 29 & $6 / 150(4.0)$ & 28.2 & 300 (median) & 10 \\
\hline Matthew T. Gettman & 13 & 215 & 35 & 0 & 22 & 170 & NR \\
\hline Sanjeev Kaul & 10 & 155 & 23 & 0 & 21 & 92 & NR \\
\hline Present study & 41 & 115 & 35 & 0 & 18.7 & 99 & 16 \\
\hline
\end{tabular}

Numbers: case numbers; time: total operation times; size: pathologic tumor size. RAPN, robot-assisted partial nephrectomy; PSM, positive surgical margin; WIT, warm ischemia time; EBL, estimated blood loss; eGFR DR, estimated glomerular filtration decrease rate (preop - postop diseased kidney); NR, no report.

seen in the operative field. However, the use of HR realtime navigation provided precise intraoperative guidelines, such as in cases where the vessels are hidden by other tissues or organs. HR can present a complete 3D image, allowing the surgeon to access the target directly and rapidly with minimal damage to surround vessels and tissues (Figures 1C,3,4). Without the support of HR, the surgeon can usually only seek out the renal artery and/or the secondary branches. However, with real-time navigation by HR, the surgeon can dissect and separate the renal vessels again, allowing for identification of the tertiary or even quaternary branch vessels. Clamping of the precise vessels supplying the tumor can effectively reduce intraoperative bleeding (Figure 3).

The aim of $\mathrm{PN}$ is the precise resection of tumors and the maximum preservation of healthy nephrons. This can be achieved by robotic surgery assisted by the HR navigation system. The robotic arm does not fatigue, does not shake, and has 360 degrees of free movement. Intraoperative realtime $3 \mathrm{D}$ imaging allows clear visualization of the tumor margins and the renal parenchyma. HR technology allows the user to rotate, magnify, and hyalinized the images surrounding the tumor margin. Even tumor margins on the opposite side or embedded deep inside tissues which cannot be visualized by the laparoscope camera, can be detected with HR (Figure 4). This allows the precise and complete removal of the tumor, enabling maximum preservation of nephrons with minimal damage.

There were several limitations to this study. First, this was a retrospective, single center study with a short followup period. Future multi-center studies with a longer follow-up period should be conducted to verify these results. Second, all the operations in our department were performed by a same surgeon and the influence of surgeons with different proficiency levels on the patient outcomes cannot be ignored.

\section{Conclusions}

The use of HR in RAPN resulted in shorter surgery time, shorter tumor resection time, and improved precision and rapid location of surgical targets. Therefore, real-time navigation with HR is a promising technology for RAPN.

\section{Acknowledgments}

Funding: None.

\section{Footnote}

Reporting Checklist: The authors have completed the STROBE reporting checklist. Available at https://dx.doi. 
org/10.21037/tau-21-473

Data Sharing Statement: Available at https://dx.doi. org/10.21037/tau-21-473

Conflicts of Interest: All authors have completed the ICMJE uniform disclosure form (available at https://dx.doi. org/10.21037/tau-21-473). The authors have no conflicts of interest to declare.

Ethical Statement: The authors are accountable for all aspects of the work in ensuring that questions related to the accuracy or integrity of any part of the work are appropriately investigated and resolved. All procedures performed in this study involving human participants were in accordance with the Declaration of Helsinki (as revised in 2013). The study was approved by ethics board of General Hospital of Central Theater Command of the People's Liberation Army (Number: [2018]012) and informed consent was taken from all the patients.

Open Access Statement: This is an Open Access article distributed in accordance with the Creative Commons Attribution-NonCommercial-NoDerivs 4.0 International License (CC BY-NC-ND 4.0), which permits the noncommercial replication and distribution of the article with the strict proviso that no changes or edits are made and the original work is properly cited (including links to both the formal publication through the relevant DOI and the license). See: https://creativecommons.org/licenses/by-nc-nd/4.0/.

\section{References}

1. Gill IS, Aron M, Gervais DA, et al. Clinical practice. Small renal mass. N Engl J Med 2010;362:624-34.

2. Lombardo R, Leonardo C, Zarraonandia A, et al. Complex renal masses: partial or no partial nephrectomy? Ann Transl Med 2019;7:S312.

3. Patel HD, Mullins JK, Pierorazio PM, et al. Trends in renal surgery: robotic technology is associated with increased use of partial nephrectomy. J Urol 2013;189:1229-35.

4. Long G, Liu M, Zhang Y, et al. Robot-assisted laparoscopic partial nephrectomy is a safe and effective option for clinical T2 renal cell carcinoma: a case-series from single-institution. Transl Cancer Res 2020;9:7140-8.

5. Boylu U, Basatac C, Yildirim U, et al. Comparison of surgical, functional, and oncological outcomes of open and robot-assisted partial nephrectomy. J Minim Access Surg 2015;11:72-7.

6. Lane BR, Gill IS. 5-year outcomes of laparoscopic partial nephrectomy. J Urol 2007;177:70-4; discussion 74.

7. Gill IS, Kavoussi LR, Lane BR, et al. Comparison of 1,800 laparoscopic and open partial nephrectomies for single renal tumors. J Urol 2007;178:41-6.

8. Rymuza B, Grodecki K, Kamiński J, et al. Holographic imaging during transcatheter aortic valve implantation procedure in bicuspid aortic valve stenosis. Kardiol Pol 2017;75:1056.

9. Tufek I, Mourmouris P, Doganca T, et al. Robot-assisted partial nephrectomy for T1b tumors: strict trifecta outcomes. JSLS 2017;21:e2016.00113.

10. Patton MW, Salevitz DA, Tyson MD 2nd, et al. Robotassisted partial nephrectomy for complex renal masses. J Robot Surg 2016;10:27-31.

11. Wang AJ, Bhayani SB. Robotic partial nephrectomy versus laparoscopic partial nephrectomy for renal cell carcinoma: single-surgeon analysis of $>100$ consecutive procedures. Urology 2009;73:306-10.

12. Benway BM, Bhayani SB, Rogers CG, et al. Robot assisted partial nephrectomy versus laparoscopic partial nephrectomy for renal tumors: a multiinstitutional analysis of perioperative outcomes. J Urol 2009;182:866-72.

13. Aron M, Koenig P, Kaouk JH, et al. Robotic and laparoscopic partial nephrectomy: a matched-pair comparison from a high-volume centre. BJU Int 2008;102:86-92.

14. Motoyama D, Matsushita $\mathrm{Y}$, Watanabe H, et al. Initial learning curve for robot-assisted partial nephrectomy performed by a single experienced robotic surgeon. Asian J Endosc Surg 2020;13:59-64.

15. Ellison JS, Montgomery JS, Hafez KS, et al. Association of RENAL nephrometry score with outcomes of minimally invasive partial nephrectomy. Int J Urol 2013;20:564-70.

16. Gettman MT, Blute ML, Chow GK, et al. Robotic-assisted laparoscopic partial nephrectomy: technique and initial clinical experience with $\mathrm{DaVinci}$ robotic system. Urology 2004;64:914-8.

17. Kaul S, Laungani R, Sarle R, et al. da Vinci-assisted robotic partial nephrectomy: technique and results at a mean of 15 months of follow-up. Eur Urol 2007;51:18691; discussion 191-2.

18. Phillips CK, Taneja SS, Stifelman MD. Robot-assisted laparoscopic partial nephrectomy: the NYU technique. J Endourol 2005;19:441-5; discussion 445. 
19. Rogers CG, Singh A, Blatt AM, et al. Robotic partial nephrectomy for complex renal tumors: surgical technique. Eur Urol 2008;53:514-21.

20. Nandurkar R, Basto M, Sengupta S. Nephron-sparing surgery for the management of upper tract urothelial carcinoma: an outline of surgical technique and review of outcomes. Transl Androl Urol 2020;9:3160-7.

21. Patard JJ, Shvarts O, Lam JS, et al. Safety and efficacy of partial nephrectomy for all T1 tumors based on an international multicenter experience. J Urol 2004;171:2181-5, quiz 2435.

22. Novick AC, Campbell SC, Belldegrun A, et al. Guideline for management for the clinical stage 1 renal mass. American Urologic Association Education and Research, Inc., 2009.

Cite this article as: Zeng S, Zhou Y, Wang M, Bao H, $\mathrm{Na}$ Y, Pan T. Holographic reconstruction technology used for intraoperative real-time navigation in robot-assisted partial nephrectomy in patients with renal tumors: a single center study. Transl Androl Urol 2021;10(8):3386-3394. doi: 10.21037/ tau-21-473
23. Lane BR, Gill IS. 7-year oncological outcomes after laparoscopic and open partial nephrectomy. J Urol 2010;183:473-9.

24. Kaouk JH, Hillyer SP, Autorino R, et al. 252 robotic partial nephrectomies: evolving renorrhaphy technique and surgical outcomes at a single institution. Urology 2011;78:1338-44.

25. Lasser MS, Doscher M, Keehn A, et al. Virtual surgical planning: a novel aid to robot-assisted laparoscopic partial nephrectomy. J Endourol 2012;26:1372-9.

26. Antonelli A, Veccia A, Palumbo C, et al. Holographic reconstructions for preoperative planning before partial nephrectomy: a head-to-head comparison with standard CT scan. Urol Int 2019;102:212-7. 